\title{
THE EFFECT OF CHRONIC VIRAL HEPATITIS B AND C ON BONE MINERAL DENSITY
}

\author{
Ecaterina-Constanta Barbu ${ }^{1,2}$, Cristina-Emilia Chitu-Tisu ${ }^{1,2}$, Mihai Lazar ${ }^{1,3}$, \\ Ramona Stefania Popescu, ${ }^{1,3}$, Adrian Octavian Abagiư ${ }^{3}$, Daniela Adriana Ion', \\ Ioana Anca Badarau ${ }^{1}$ \\ 1 "Carol Davila" University of Medicine and Pharmacy, Bucharest, Romania \\ 2 "Dr. I. Cantacuzino" Clinical Hospital, Bucharest, Romania \\ 3 “Prof. Dr. Matei Bals” National Institute for Infectious Diseases, Bucharest, Romania
}

\begin{abstract}
Objectives. Chronic viral hepatitis B and C represent an important health burden all over the world. Reduced bone mineral density is an extrahepatic complication which has been found in patients with chronic liver disease. The aim of our study was to identify bone mineral impairment (osteopenia/osteoporosis) and the risk factors that are correlated with its severity, in patients with chronic viral hepatitis $B(\mathrm{CHB})$ and $\mathrm{C}(\mathrm{CHC})$.

Material and methods. Anthropometric, biological parameters and bone mineral density (BMD) were measured in 60 patients with CHB $(n=30)$ and CHC $(n=30)$. BMD was assessed using Dual Energy X-ray Absorptiometry (DEXA) in the hip and lumbar spine regions, inclusively a whole scan (total body).

Results. Sixty patients (mean age 44.93 years, range: 20-70) were enrolled, including $30 \mathrm{CHB}$ patients (mean age 46.43 years, range: $20-70$ ) and $30 \mathrm{CHC}$ patients (mean age 43.43 years, range: $28-64$ ). Forty of patients were men $(66.66 \%)$. Active smokers were 16 patients $(26.66 \%)$. Meanbody mass index (BMI) was $25.38 \mathrm{~kg} / \mathrm{m}^{2}$ (range: 16.70-38.40). At baseline, 21 of 60 (35\%) of the patients had evidence of osteopenia and 4 of $60(6.66 \%)$ of patients, respectively presented osteoporosis at LS. At total hip, 22 of 60 of the patients $(36.66 \%)$ recorded osteopenia; osteoporosis was found at 7 patients $(11.66 \%)$ at total hip assessment. Low BMD values at different regions correlated significantly with low BMI, smoking and liver fibrosis grade.

Conclusions. Our results suggest that bone mineral metabolism disorders exist in patients with chronic viral hepatitis B and C who are active smokers, presenting low BMI and advanced liver fibrosis, even without liver cirrhosis.
\end{abstract}

Keywords: osteodystrophy, osteoporosis, chronic viral hepatitis B, chronic viral hepatitis C, chronic liver disease, bone mineral density

\section{INTRODUCTION}

Reduced bone mineral density, as an extrahepatic complication, found in patients with chronic liver disease (CLD) is well known (1-3). This complication was defined as hepatic osteodystrophy (HO) (3) and commonly manifests as osteoporosis or osteopenia (4). The pathophysiology of this type of secondary osteoporosis still remains unclear (3). Many factors seem to be evolved in the occurrence of HO: chronic cholestasis (5) (especially in patients with primary biliary cirrhosis), low levels of insulin-like growth factor-1 (IGF-1) $(6,7)$, the af- fecting of system of the receptor activator of nuclear factor кappa B ligand (RANKL) and osteoprotegerin (OPG) (8), vitamin D deficiency $(9,10)$, sedentary lifestyle (11), low body mass index (BMI) (12), alcoholism (13), and the list is still opened. The prevalence of $\mathrm{HO}$ ranges from 12 to $55 \%$ in patients with chronic liver disease (3).

Regarding the chronic viral hepatitis, the prevalence (in Europe) was reported to be between 0.1$7 \%$ for chronic hepatitis B and between $0.4-3.5 \%$ in general population for chronic hepatitis $\mathrm{C}$, respectively (14). 
In chronic viral hepatitis, an inflammatory response dominates the pathophysiologic response to infection with an increase release of cytokines (increased osteoclast bone resorption) $(15,16)$. The risk of $\mathrm{HO}$ development increases as a result of diagnosis of chronic viral hepatitis $\mathrm{B}$ or $\mathrm{C}$ at young ages and additionally, as the lifespan of these patient increases due to new antiviral therapy. Although, there is much interest in this field, little is known about this pathology so that frequently remains underdiagnosed and therefore untreated. The management of this type of osteoporosis rises many problems, especially in patients with liver cirrhosis and so far it has not been established a specific treatment.

\section{OBJECTIVES}

Considering all the above aspects in this pathology, the aim of our study was to identify bone mineral impairment (osteopenia/osteoporosis) and the factors that are correlated with its severity, in patients with chronic viral hepatitis B and C.

\section{MATERIAL AND METHODS}

Sixty patients with chronic viral hepatitis B and C (30 with CHB, 30 with $\mathrm{CHC}$ ), from "Prof. Dr. Matei Bals" National Institute for Infectious Diseases, Bucharest were included in this cross-sectional study. All patients were enrolled between
July 2014 and January 2015. The study protocol was approved by the Institute Ethics Committee. The relevant demographic (age, gender), anthropometric (height, weight, body mass index - BMI), smoker status data and disease related data (liver function test - liver enzymes), HBV-DNA (Deoxyribonucleic acid) and HCV-RNA [(Ribonucleic acid) by quantitative polymerase chain reaction PCR] are filled out in Table 1.

We calculated BMI as weight $(\mathrm{kg}) /$ height $\left(\mathrm{m}^{2}\right)$ and defined it according to WHO criteria (17). For the non-invasive assessment ofliver fibrosis we used FibroTest (FT, BioPredictive, Paris, France), a non-invasive marker to stage liver fibrosis (18).

All patients were aged over 18 years and had persistently (more than 6 months) positive serological markers - hepatitis B surface antigen/HBVDNA, antibodies HCV/HCV-RNA and/or histological diagnosis of $\mathrm{HBV} / \mathrm{HCV}$ infection. All women included in the study were premenopausal. Every patient read and signed the informed consent before enrolling to the study (Inclusion criteria).

Menopause (physiological/surgical), HIV coinfection, cirrhosis, autoimmune liver disease, bone disorders, endocrynopathies (inclusively diabetes mellitus), vitamin D deficiency, severe heart disease, history of malignancy, chronic kidney disease (estimated glomerular filtration rate $<50 \mathrm{ml} /$ $\mathrm{min} / 1.73 \mathrm{~m}^{2}$ ), cortisone therapy $>3$ months, treatment for osteoporosis, prolonged immobilization, malnutrition, examination with iodinated contrast agents in the last 72 hours before DEXA assess-

TABLE 1. The demographic, anthropometric and biological parameters in hepatitis $B$ and $C$ patients

\begin{tabular}{|c|c|c|c|}
\hline Parameter & All patients $(n=60)$ & Hepatitis B $(n=30)$ & Hepatitis C $(n=30)$ \\
\hline Age (yr) & $44.93(20-70)$ & $46.43(20-70)$ & $43.43(28-64)$ \\
\hline Male, n (\%) & $40(66.66)$ & $23(76.66)$ & $17(56.66)$ \\
\hline Female, n (\%) & $20(33.34)$ & $7(23.34)$ & $13(43.34)$ \\
\hline BMI $\left(\mathrm{kg} / \mathrm{m}^{2}\right)$ & $25.38(16.70-38.40)$ & $25.80(18.30-35.12)$ & $24.96(16.70-38.40)$ \\
\hline Smoking, n (\%) & $16(26.66)$ & $4(13.33)$ & $12(40)$ \\
\hline $\begin{array}{c}\text { HBV-DNA (IU/mL) } \\
\text { Undetectable } \\
\text { Detectable } \\
\text { Mean HBV-DNA }\end{array}$ & & $\begin{array}{c}15(50 \%) \\
15(50 \%) \\
9519955.53 \\
(20-100000000) \\
\end{array}$ & \\
\hline $\begin{array}{c}\text { HCV-RNA (IU/mL) } \\
\text { Undetectable } \\
\text { Detectable } \\
\text { Mean HCV-RNA }\end{array}$ & & & $\begin{array}{c}13(43.33) \\
17(56.67) \\
929086.82 \\
(4142-3200000) \\
\end{array}$ \\
\hline $\begin{array}{c}\text { Fibrotest, n (\%) } \\
\text { F0 } \\
\text { F1 } \\
\text { F2 } \\
\text { F3 } \\
\end{array}$ & $\begin{array}{c}9(15) \\
7(11.66) \\
22(36.67) \\
22(36.67) \\
\end{array}$ & $\begin{array}{c}4(13.33) \\
3(10) \\
10(33.33) \\
13(43.34) \\
\end{array}$ & $\begin{array}{c}5(16.67) \\
4(13.33) \\
12(40) \\
9(30) \\
\end{array}$ \\
\hline GPT (IU/mL) & $68.14(11-754)$ & $81.67(11-754)$ & $54.6(18-184)$ \\
\hline
\end{tabular}

BMI - Body Mass Index; GPT - Glutamate-Pyruvate Transaminase; HBV-DNA - Hepatitis B Virus Deoxyribonucleic acid; HCV-RNA - Hepatitis C Virus Ribonucleic acid; HBV - Hepatitis B virus; HCV - Hepatitis C virus; $\mathrm{IU} / \mathrm{mL}$ - International Units/Milliliter; $\mathrm{n}$ - Number; $\mathrm{Yr}$ - years. 
ment were the exclusion criteria. There was no family history of osteoporosis.

None of the patients received any treatment related with bone metabolism (calcium, vitamin D, corticosteroids, hormone replacement therapy). No patient had personal history of bone fracture at the beginning of the research.

\section{BONE DENSITY MEASUREMENTS}

Bone mineral density was assessed in all patients at the lumbar spine (LS, L1-L4), at the total hip and also at whole skeleton (total body) using DEXA (Unit bone densitometry (DEXA) - GE LUNAR DPX-NT), which is the gold standard test in diagnosis of osteoporosis (19). According to World Health Organization (19) recommendations, we used T-score (patient BMD compared to the BMD of a young adult reference population of the same sex) and Z-score (patient BMD compared to the BMD of an age, sex, ethnicity-matched reference population). All scans were carried out on the same machine and were analyzed with the same software program. Osteopenia was defined as T-score between -1 and -2.5 standard deviations (SD) and osteoporosis as $\mathrm{T}$-score $\leq-2.5 \mathrm{SD}$ below the mean BMD for young adults. A Z-score $\leq-2$ defined low BMD for chronological age ("below the expected range for age").

\section{STATISTICAL ANALYSES}

In the present research, data were expressed as median and range for continuous variables and frequency counts for categorical variables. We used Fisher's exact test for binary variables, the MannWhitney U test and the Kruskal-Wallis test for continuous variables, in order to evaluate the differences between groups. A p-value less than 0.05 was considered significant.

\section{RESULTS}

The demographic, anthropometric, biological parameters in the study patients are listed in Table 1. A total of 60 patients (mean age 44.93 years, range: 20-70) were enrolled, including $30 \mathrm{CHB}$ patients (mean age 46.43 years, range: $20-70$ ) and $30 \mathrm{CHC}$ patients (mean age 43.43 years, range: $28-64$ ). Forty of patients were men $(66.66 \%)$. Active smokers were 16 patients $(26.66 \%)$. Mean BMI was 25.38 $\mathrm{kg} / \mathrm{m}^{2}$ (range: $16.70-38.40$ ).

Regarding the liver enzymes test, the mean glutamate-pyruvate transaminase (GPT) did not differ significantly between HBV and HCV patients (Table 1) and did not correlated with low BMD. About half of patients, in similar proportions for the two groups, had serum undetectable viral loads. The majority of the patients (44 patients, 73.34\%) had F2 and F3 stages at the non-invasive assessment of liver fibrosis (using FibroTest).

The mean T-scores at total body assessment were $0.44(-2.3-2.4)$ for CHB patients and -0.01 $(-2.6-3)$ for CHC patients. At LS and total hip assessment, the mean T-scores were $-0.74(-2.4-$ $1.4)$ and $-0.19(-2.3-2)$, respectively for CHB patients; $-0.7(-2.8-2.2)$ and $-0.8(-2.7-1.9)$, respectively for $\mathrm{CHC}$ patients. At baseline, 21 of 60 $(35 \%)$ of the patients had evidence of osteopenia and 4 of $60(6.66 \%)$ of patients, respectively presented osteoporosis at LS. At total hip, 22 of 60 of the patients $(36.66 \%)$ recorded osteopenia; osteoporosis was found at 7 patients $(11.66 \%)$ at total hip assessment. The overall data of BMD measurements are found in Table 2.

TABLE 2. DEXA scan assessment in hepatitis $B$ and $C$ patients

\begin{tabular}{|l|c|c|c|}
\hline Parameter & $\begin{array}{c}\text { All patients } \\
\text { ( } \mathbf{n = 6 0} \text { ) }\end{array}$ & $\begin{array}{c}\text { Hepatitis B } \\
\text { ( } \mathbf{n = 3 0 )}\end{array}$ & $\begin{array}{c}\text { Hepatitis C } \\
\text { ( } \mathbf{n = 3 0 )}\end{array}$ \\
\hline Osteopenia, n (\%) & $28(46.66)$ & $15(50)$ & $13(43.33)$ \\
Total body & $9(15)$ & $5(16.66)$ & $4(13.33)$ \\
Lumbar spine, L1-L4 & $21(35)$ & $11(36.66)$ & $10(33.33)$ \\
Total hip & $22(36.66)$ & $11(36.66)$ & $11(36.66)$ \\
\hline Osteoporosis, n (\%) & $7(11.66)$ & $4(13.33)$ & $3(10)$ \\
Total body & $3(5)$ & $1(3.33)$ & $2(6.66)$ \\
Lumbar spine, L1-L4 & $4(6.66)$ & $2(6.66)$ & $2(6.66)$ \\
Total hip & $7(11.66)$ & $4(13.33)$ & $3(10)$ \\
\hline
\end{tabular}

DEXA - Dual Energy X ray Absorptiometry; $\mathrm{n}$ - Number

We found that low BMD values (T-scores) significantly correlated with low BMI values at total hip $(\mathrm{p}=0.001)$. Patients that are active smokers presented lower T-scores than non-smokers and smoking significantly associated with low BMD at total hip. $(\mathrm{p}=0.027)$. When we classified patients according to fibrosis grade, the BMD at LS and total hip was lower in more advanced stages of liver disease $(p=0.031$ and $p=0.010)$ for both groups. The T-scores and BMD values were lower in $\mathrm{CHC}$ patients than in the CHB ones but there was no significantly correlation at $\operatorname{LS}(\mathrm{p}=0.333)$ and total hip $(p=0.223)$, although at total body assessment $p$ value is at the limit at significance $(p=0.048)$. Median T-scores did not differ between the genders not only at LS but also at total hip $(\mathrm{p}=0.601$ and $\mathrm{p}=0.723$ ). There was no correlation found between $\mathrm{Z}$-score values and age, gender, BMI, smoking, liver function or fibrosis grade. 


\section{DISCUSSIONS}

The effects of chronic viral hepatitis B and Con bone mineral metabolism represent an important issue in the medical research field in the last years.

In our study, the prevalence of low BMD in patients with chronic viral hepatitis $\mathrm{B}$ and $\mathrm{C}$ was high (28 of patients $-46.66 \%$ - were osteopenic and $7-$ $11.66 \%$ - of patients were osteoporotic). These results are in accordance with the results of other studies (20). We obtained important proportions of low BMD in each site of the skeleton although the mean $\mathrm{BMD}$ and the $\mathrm{T}$ - and $\mathrm{Z}$-scores were in normal ranges.

The patients from the two groups had mean BMI ranged in the overweight category; although, we reported that low BMD values were significantly associated with low BMI values at total hip assessment. This fact that was reported also by other authors (21).

It is well known that the current bone mass is determined by genetic factors but also by lifestyle factors, including cigarettes smoking (22). Our finding that active smoking correlated significantly with bone demineralization confirmed once again smoking bad effect on mineral metabolism. There are many studies that have contributed to this fact $(21,23,24)$.

Schiefke et al. reported in their research that patients with more advanced stages of liver disease (histologically proven) presented lower BMD (20). In accordance to their research, in our study patients who had advanced stage liver disease presented more demineralization at LS and total hip than the ones with mild fibrosis.

We did not achieved any correlation between the gender of the patient and the BMD values in both groups of patients. Lucaci et al., found that BMD disorders were more important in women than in men (25). Also, in this research, age was not found as a predictive risk factor. On the contrary, Lucaci et al., reported an important influence of age on BMD (25); but Orsini et al. was in accordance with our results regarding this risk factor (21).

As limitations of this study, we mention: the small lot size, the cross-sectional study design (a prospective study design it would evaluate better the correlation for the predictive risk factors).

\section{CONCLUSION}

Our results suggest that bone mineral metabolism disorders do exist in patients with chronic viral hepatitis $\mathrm{B}$ and $\mathrm{C}$ who are active smokers, presenting low BMI and advanced liver fibrosis, even without liver cirrhosis.

Extrapolating the results of our study and considering the anterior studies in the field, we may be able to see the important social and economic burden of this pathology together with its consequences - the fractures. Having this in mind, we need to focus on larger studies, with prospective design that should contribute to the understanding of this disease and its modality of management.

\section{Acknowledgement}

"This paper is supported by the Sectorial Operational Program Human Resources Development (SOP HRD), financed by the European Social Fund and by the Romanian Government under the contract number POSDRU/159/1.5/S/137390“.

\section{REFERENCES}

1. Rouillard S., Lane N.E. Hepatic osteodystrophy. Hepatology 2001; 33:301-7.

2. Collier J.D., Ninkovic M., Compston J.E. Guidelines on the management of osteoporosis associated with chronic liver disease. Gut 2002; 50 Suppl 1:i1-9.

3. Lopez-Larramona G., Lucendo A.J., Gonzalez-Castillo S., Tenias J.M. Hepatic osteodistrophy: An important matter for consideration in chronic liver disease. World J Hepat. 2013; 3:300-7.

4. Marignani M., Angeletti S., Capurso G., etc. Bad to the bone: the effects of liver diseases on bone. Minerva Med 2004; 95:489-505.

5. Gasser R.W. Cholestasis and metabolic bone disease - a clinical review. Wien Med Wochenschr 2008; 158:553-7.

6. Goral V., Simsek M., Mete N. Hepatic osteodystrophy and liver cirrhosis. World J Gantroenterol 2010; 16:1639-1643.

7. Gallego-Rojo F.J., Gonzalez-Calvin J.L., Munoz-Torres M., etc Bone mineral density, serum insulin-like growth factor 1 and bone turnover markers in viral cirrhosis. Hepatology 1998; 28:695-9.

8. Gaudio A., Lasco A., Morabito N., etc. Hepatic osteodystrophy: does the osteoprotegerin/receptor activator of nuclear factor-kB ligand system play a role? J Endocrinol Invest 2005; 28:677-82.

9. Arteh J., Narra S., Nair S. Prevalence of vitamin D deficiency in chronic liver disease. Dig Dis Sci 2010; 55:2624-8.

10. Crawford B.A., Labio E.D., Strasser S.I., McCaughan G.W. Vitamin D replacement for cirrhosis-related bone disease. Nat Clin Pract Gastroenterol Hepatol 2006; 3:689-99.

11. Hay J.E., Guichelaar M.M. Evaluation and management of osteoporosis in liver disease. Clinics in liver disease 2005; 9:747-66.

12. Mounach A., Ouzzif Z., Wariaghli G., etc. Primary biliary cirrhosis and osteoporosis: a case-control study. Journal of bone and mineral metabolism 2008; 26:379-84.

13. Kim M.J., Shim M.S., Kim M.K., etc. Effect of chronic alcohol ingestion on bone mineral density in males without liver cirrhosis. Korean J Intern Med 2003; 18:174-80. 
14. European Centre for Disease Prevention and Control. Hepatitis $B$ and $\mathrm{C}$ in the EU neighbourhood: prevalence, burden of disease and screening policies. Stockholm: ECDC2010.

15. Nakchbandi I.A. Osteoporosis and fractures in liver disease: relevance, pathogenesis and therapeutic implications. World $\mathrm{J}$ Gastroenterol 2014; 20:9427-38.

16. Corazza G.R., Trevisani F., Di Stefano M., etc. Early increase of bone resorption in patients with liver cirrhosis secondary to viral hepatitis. Dig Dis Sci 2000; 45:1392-9.

17. World Health Organization (2000) Obesity. Preventing and Managing the Global Epidemic. Technical Report Series No. 894 WHO G, 2000.

18. Imbert-Bismut F.R.V., Pieroni L., etc. Biochemical markers of liver fibrosis in patients with hepatitis $C$ virus infection: a prospective study. Lancet 2001; 357:1069-107.

19. Assessment of fracture risk and its application to screening for postmenopausal osteoporosis. Report of a WHO Study Group. World Health Organ Tech Rep Ser 1994; 843:1-129.

20. Schiefke I., Fach A., Wiedmann M., etc. Reduced bone mineral density and altered bone turnover markers in patients with non- cirrhotic chronic hepatitis B or C infection. World J Gastroenterol 2005;11:1843-7.

21. Orsini L.G.S., Pinheiro M.M., Castro C.H.M., etc. Bone mineral density measurements, bone markers and serum vitamin $D$ concentrations in men with chronic non-cirrhotic untreated hepatitis C. PLOS One 2013; 8:e81652.

22. Felecia C., David D. Pathogenesis of osteoporosis. Rheumatology. Mosbey: Elsevier 2011.

23. Yenice N., Gumrah M., Mehtap O., etc. Assessment of bone metabolism and mineral density in chronic viral hepatitis. Turk $\mathrm{J}$ Gastroenterol 2006; 17:260-6.

24. Nanda K.S., Ryan E.J., Murray B.F., etc. Effect of chronic hepatitis $C$ virus infection on bone disease in postmenopausal women. Clinical Gastroenterology and Hepatology 2009; 7:894-899.

25. Lucaci C., Acalovschi M. Hormonal and Cytokine Implications in the Pathophysiology of Osteoporosis Occurring in Chronic Liver Diseases. Maedica (Buchar) 2012; 7:358-63. 